\title{
Solid-solid collapse transition in a two dimensional model molecular system
}

\author{
Rakesh S. Singh and Biman Bagch* \\ Solid State and Structural Chemistry Unit, Indian Institute of Science, Bangalore 560012, India
}

(Dated: March 6, 2018)

\begin{abstract}
Solid-solid collapse transition in open framework structures is ubiquitous in nature. The real difficulty in understanding detailed microscopic aspects of such transitions in molecular systems arises from the interplay between different energy and length scales involved in molecular systems, often mediated through a solvent. In this work we employ Monte Carlo (MC) simulations to study the collapse transition in a model molecular system interacting via both isotropic as well as anisotropic interactions having different length and energy scales. The model we use is known as Mercedes-Benz (MB) which for a specific set of parameters sustains three solid phases: honeycomb, oblique and triangular. In order to study the temperature induced collapse transition, we start with a metastable honeycomb solid and induce transition by heating. High density oblique solid so formed has two characteristic length scales corresponding to isotropic and anisotropic parts of interaction potential. Contrary to the common believe and classical nucleation theory, interestingly, we find linear strip-like nucleating clusters having significantly different order and average coordination number than the bulk stable phase. In the early stage of growth, the cluster grows as linear strip followed by branched and ring-like strips. The geometry of growing cluster is a consequence of the delicate balance between two types of interactions which enables the dominance of stabilizing energy over the destabilizing surface energy. The nuclei of stable oblique phase are wetted by intermediate order particles which minimizes the surface free energy. We observe different pathways for pressure and temperature induced transitions.
\end{abstract}

\section{INTRODUCTION}

Several studies have shown that many solids exhibit temperature and pressure induced solid-solid phase transitions resulting in a discontinuous change in material properties [1 7 ]. In the case of shock wave induced solidsolid transitions both temperature and pressure of the system increases [4, 5]. The well known examples of solidsolid transition are $\mathrm{Fe}$ and its alloys ( $\mathrm{Fe} / \mathrm{Ni}$ alloys transform on cooling from face-centered cubic (fcc) phase to a low-temperature body-centered cubic (bcc) phase), alloys based on $\mathrm{CuAl}, \mathrm{NiTi}, \mathrm{NiAl}$, and oxide ceramics (like $\mathrm{ZrO} 2$ ), name only a few. In steel, the structural transitions are used to enhance its strength. Carbon crystallizes in the hexagonal graphite structure at ambient pressure and undergoes a pressure-induced transition to the tetrahedral diamond structure. Structural transformations are also observed in biological systems. Some virus species use the pressure-induced martensitic transformation to infect bacteria cells[1]. Another application of solid-solid transition is the shape-memory effect in many alloys and widely used in medicinal science [3]. Solid-solid transitions can be driven either by homogenous or heterogeneous (in the case of presence of preexisting defects which reduces the free energy barrier for transition) nucleation. Despite the great importance of solid-solid transitions in material, medicinal and biological sciences, little is known about the microscopic aspects of solid-solid transitions. In molecular systems, the real difficulty in understanding detailed microscopic aspects of such transitions arises from the interplay between dif-

\footnotetext{
* Corresponding author: bbagchi@sscu.iisc.ernet.in
}

ferent energy and length scales involved and often mediated through a solvent.

Computer simulations are the most appropriate technique to study the microscopic aspects of solid-solid transitions. However, the difficulty in computer simulations arises in construction of potential which can give rise to multiple solid phases. Now it is well accepted that systems interacting with core soft potentials show rich phase diagram consisting multiple solid phases [8-12]. Coresoftened potential was first used by Hemmer and Stell [8] in a lattice gas system to discuss the isostructural solidsolid phase transition. Later, Young and Alder 9] showed that a two dimensional system interacting via repulsive step potential (hard-core plus square-shoulder repulsion) can rise to phase diagram and solid-solid phase transitions qualitatively similar to those observed in cesium and cerium. Distinct solid phases in systems interacting with core soft isotropic potential arise due to the presence of distinct length scales in the potential. Recently, Sengupta and coworker [13] introduced a potential where particles interact via modified Lennard-Jones potential and each particle has two internal states. They also observed a rich phase diagram showing water-like thermodynamic anomalies and polymorphism. Although there are many computer simulation studies that discuss the rich phase diagram consisting many solid phases [8 14$]$ only few studies are devoted for understanding of nature and pathway of solid-solid transitions. Recently, Rao and Sengupta [15 17] studied the nucleation of a triangular phase from bulk metastable square crystal in a model two dimensional system interacting via both two and three body potentials. These studies suggest that at high temperature the critical nucleus of the triangular phase is isotropic and at low temperature the critical nucleus is anisotropic twinned bicrystal. They also observed that 
the final microstructures depend on quench rate. For systems like iron the dependence of microstructures on rate of cooling (quench depth) is well known. Slow cooling rate results in an equilibrium ferrite phase while rapid cooling results the trapping of system in a metastable twinned martensite.

In this work we have studied the microscopic pathway for the solid-solid collapse transition in a model two dimensional (2D) molecular system interacting via both isotropic as well as anisotropic interactions having different length and energy scales. The model we use is known as Mercedes-Benz (MB) potential [18 20] which on specific parameterization for relative strength of isotropic interaction sustains three solid phases: honeycomb, oblique and triangular 21]. The complexity of MB model and simplicity of $2 \mathrm{D}$ allows us to explore several aspects of solid-solid transitions not observed in atomic systems. In the subsequent sections we shall discuss the honeycomb to oblique solid collapse transition.

The organization of the rest of the paper is as follows. In the next section (Section II), we present the model system and the simulation details used in this work. In Sections III, first, we discuss the free energy surface and then the microscopic pathway for temperature and pressure induced honeycomb to oblique solid transition. In Section IV we present concluding remarks.

\section{MODEL AND SIMULATION DETAILS}

Mercedes-Benz potential has two terms - isotropic Lennard-Jones (LJ) and an anisotropic hydrogen bond (HB) term written in a completely separable form as 18 20],

$$
U\left(X_{i}, X_{j}\right)=U_{L J}\left(r_{i j}\right)+U_{H B}\left(X_{i}, X_{j}\right),
$$

where $X_{i}$ denotes vectors representing both the coordinates and orientation of the $i^{t h}$ particle and $r_{i j}$ the distance between the centers of two molecules. $U_{L J}$ is defined as

$$
U_{L J}\left(r_{i j}\right)=4 \varepsilon_{L J}\left[\left(\sigma_{L J} / r_{i j}\right)^{12}-\left(\sigma_{L J} / r_{i j}\right)^{6}\right],
$$

where $\varepsilon_{L J}$ and $\sigma_{L J}$ are the wel-depth and diameter for the isotropic LJ interaction. The anisotropic HB part, $U_{H B}$, is defined as

$$
\begin{aligned}
& U_{H B}\left(X_{i}, X_{j}\right)=\varepsilon_{H B} G\left(r_{i j}-\sigma_{H B}\right) \\
& \times \sum_{k, l=1}^{3} G\left(i_{k} \cdot u_{i j}-1\right) G\left(j_{l} \cdot u_{i j}+1\right),
\end{aligned}
$$

where $G(x)=\exp \left(-x^{2} / 2 \sigma^{2}\right)$ with $\sigma=0.085$. The unit vector $i_{k}$ represents the $k^{\text {th }}$ arm of the $i^{\text {th }}$ particle $(k=$ $1,2,3)$ and $u_{i j}$ is the unit vector joining the center of particle $i$ to the center of particle $j$. The HB parameters are $\varepsilon_{H B}=-1$ and bond length $\sigma_{H B}=1$. LJ contact distance $\left(\sigma_{L J}\right)$ is $0.7 \sigma_{H B}$. Anisotropic parameter which is a measure of relative strength of the isotropic to anisotropic interaction is defined as $\lambda_{i}=\varepsilon_{L J} / \varepsilon_{H B}$. In the present study we have used $\lambda_{i}=0.4$. Note that we have two length as well as energy scales corresponding to the isotropic LJ and anisotropic HB interactions. Monte Carlo simulations are performed in constant pressure and temperature (NPT) ensemble having number of particles $N=576,836$ and 1008. All quantities are represented in reduced units scaled with HB parameters such as temperature as $k_{B} T /\left|\varepsilon_{H B}\right|$ and distance is scaled with hydrogen-bond length, $\sigma_{H B}$.

In the case of temperature induced transition (except Fig. 1C) we started with honeycomb structure at $T=0.05$ and then increased the temperature in steps of 0.01 up to $T=0.10$ and then increased in steps of 0.0005 at $P$ $=0.10$. We first equilibrated the system for $5 \times 10^{6} \mathrm{MC}$ steps and then collected the trajectories for next $5 \times 10^{6}$ MC steps. For pressure induced transition we started with honeycomb lattice at pressure $P=0.10$ and then increased the pressure in steps of 0.05 up to $P=0.4$ and then increased in steps of 0.01 at $T=0.05$. In this case the equilibration step was $1.5 \times 10^{7} \mathrm{MC}$ steps and then collected the trajectories for next $1 \times 10^{7} \mathrm{MC}$ steps.

\section{RESULTS AND DISCUSSION}

\section{A. Free energy surface}

As the form of the Mercedes-Benz potential is written as a completely separable isotropic and anisotropic parts having distinct ground states (triangular and honeycomb), one would expect that the interplay between the relative strength of the isotropic to anisotropic interaction can give rise distinct solid phases - either stable or metastable. Recently, we find that the interplay between the strength of isotropic and anisotropic interactions gives rise to rich phase diagram[21]. The computed phase diagram consists of isotropic liquid and three crystalline phases - honeycomb, oblique and triangular. Interestingly, we note that the oblique phase is neither the ground state of isotropic nor the anisotropic part of the potential. It forms due to delicate balance between the two types of interactions. In order to study the solidsolid collapse transition from low density open structured honeycomb to high density oblique phase the honeycomb solid (ground state of the anisotropic part of the potential) should be metastable (as shown in Fig. 1A) at a given temperature, pressure and relative strength of isotropic interaction $\left(\lambda_{i}\right)$. We have constructed such a free energy surface by tuning the relative strength of the isotropic interaction $\left(\lambda_{i}\right)$.

The honeycomb lattice is stabilized by 3 hydrogen bonds (HBs) per particle and the oblique phase is stabilized by two HBs per particle along with LJ interactions (see Fig. 1B). Alignment of bonds in Fig. 1B indicates hydrogen bonding. Since both the phases are solid and stabilized by anisotropic interactions (either com- 

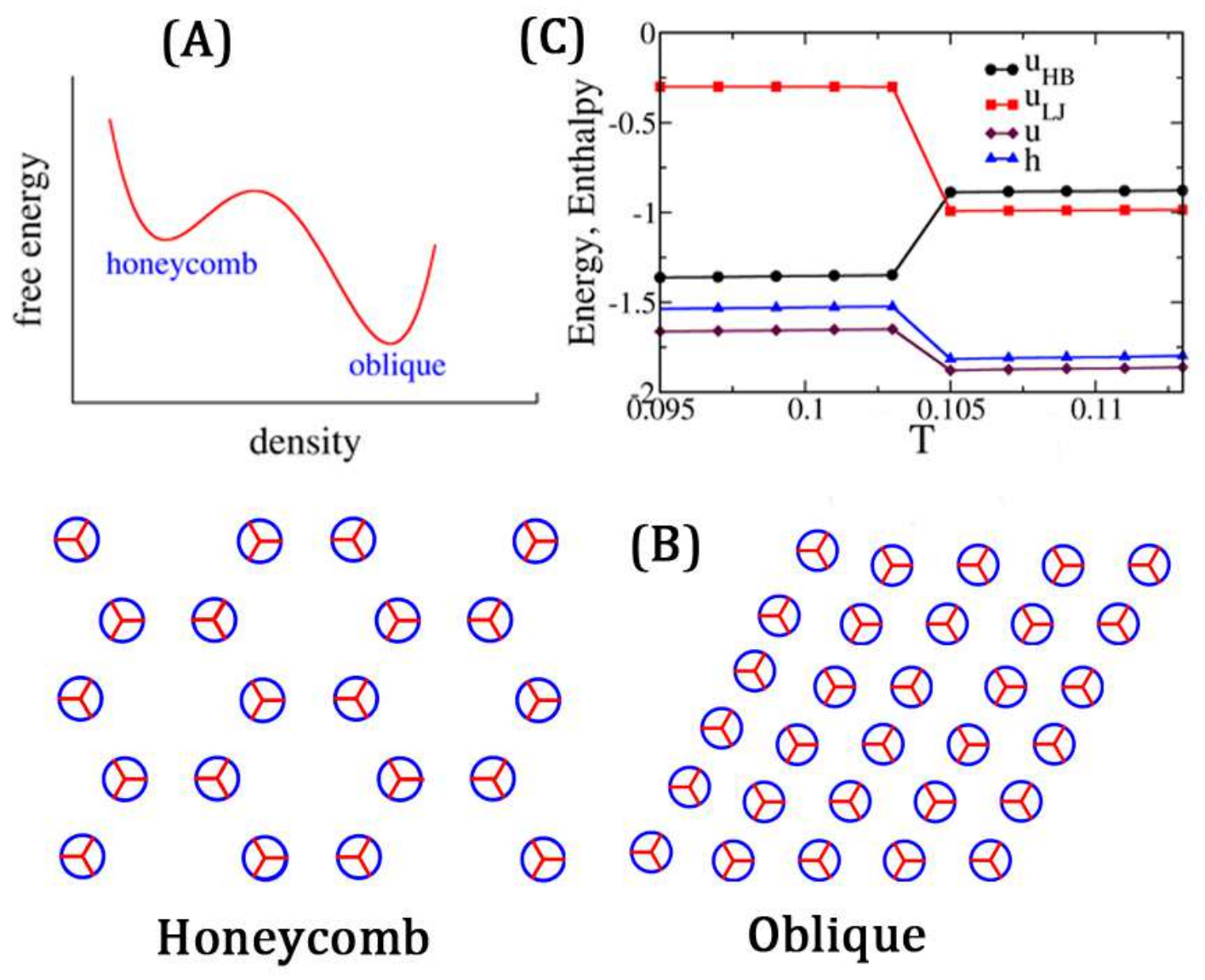

FIG. 1. (A) The proposed free energy surface for honeycomb to oblique solid-solid transition at $\lambda_{i}=0.4$ is shown. (B) The structures of the honeycomb and oblique solid phases are shown. Alignment of bonds indicates hydrogen bonding. (C) Isotropic $\left(u_{L J}\right)$ and anisotropic $\left(u_{H B}\right)$ interaction energies per particle and the total energy per particle $(\mathrm{u})$ before and after transition are shown. Blue line (with triangle up symbol) indicates the dependence of enthalpy change per particle (h) on temperature. Note the very weak temperature dependence of energy/enthalpy and switch in the contributions of isotropic and anisotropic energies to the total energy on transition.

pletely or partially), one would expect that entropy of two phases will not differ significantly. Only vibrational entropy (and some contribution from mobility of defects) is present and also not expected to differ significantly as both the solid phases are stabilized by anisotropic interactions, though, the fraction of directional bonding decreases on going from honeycomb to oblique phase. This indicates that entropy has very little role (compared to gas-liquid and liquid-solid transitions) in the construction of free energy surface for solid-solid transitions. Thus the temperature change should not alter significantly the free energy surface; however, it is important to overcome the required free energy barrier for the transition.

To support the proposed free energy surface (Fig. 1A) in Fig. 1C, we have shown the temperature dependence of enthalpy, energy and relative contribution of the isotropic (LJ) and anisotropic (HB) interactions to the total energy per particle of the system for isotropic to anisotropic strength ratio, $\lambda_{i}=0.4$. The starting configuration is honeycomb lattice at pressure $P=0.10$ and $T=0.05$, which on heating collapses to the high density oblique phases. Although, in Fig. 1C we have shown the dependence of energy and enthalpy on temperature near the transition temperature only, we find that very weak dependence persists up to much lower temperature $T=$ 0.05. Note that, contrary to the ordinary solid-liquid transition (where total energy of the system increases), in this case the total energy of the system decreases. A very weak dependence of enthalpy on temperature and decrease in the enthalpy of the system on transition supports the free energy surface proposed in Fig. 1A. Honeycomb solid is metastable at given initial pressure and temperature $(T=0.05$ and $P=0.10)$. We also note that the relative contributions of LJ and HB parts of interaction potential to the total energy per particle is switching. This indicates the increased importance of isotropic interaction in the oblique solid phase.

In order to gain quantitative insight into the structures of honeycomb and oblique phases, in Fig. 2 we have shown the computed radial distribution functions 

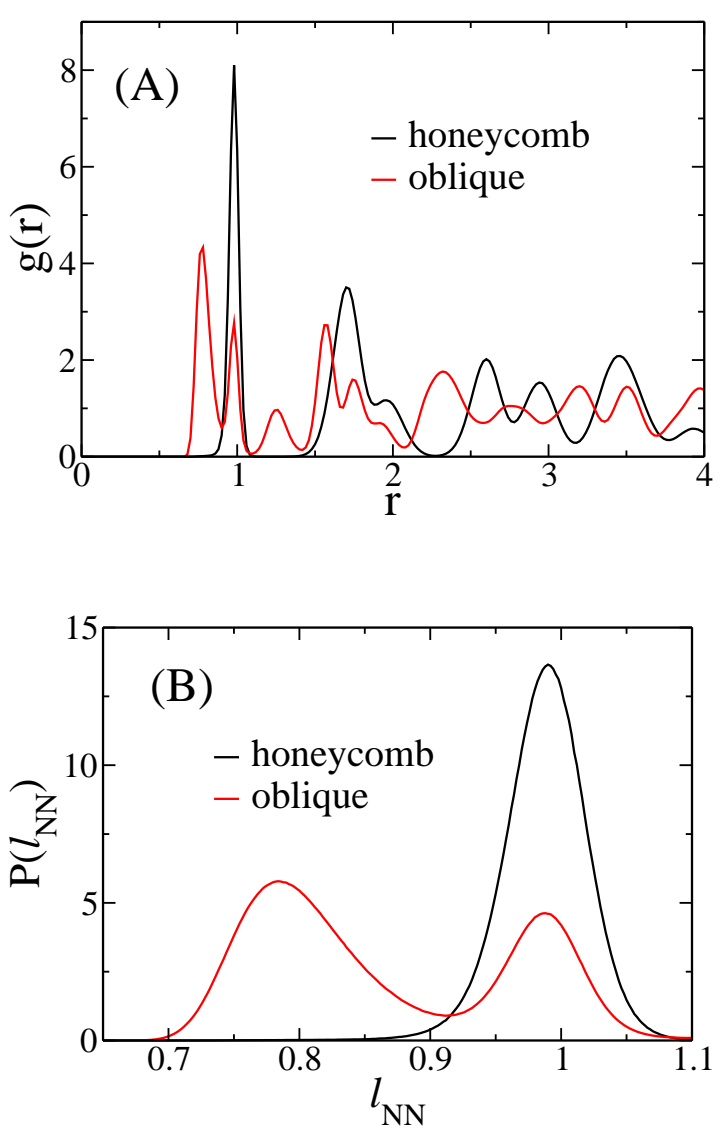

FIG. 2. (A) Radial distribution functions for honeycomb and oblique solid phases are shown. (B) Bond length distributions of nearest neighbors for honeycomb and oblique solid phases are shown. Note the splitting of first peak of radial distribution function for the oblique and also the bimodal bond length distribution of nearest neighbors. The splitting of the $1^{\text {st }}$ peak of radial distribution function and bimodal distribution of nearest neighbor bond length distribution suggest that the oblique solid has two characteristic length scales corresponding to hydrogen-bond and vander Waals (LJ) interactions.

and bond length distributions of nearest neighbors for both honeycomb and oblique solid phases near transition temperature. We have used the Voronoi construction in order to identify the nearest neighbors $(N N)$ of each particle. Note the splitting of first peak of radial distribution function and also the bimodal bond length distribution of nearest neighbors for the oblique solid phase. This signifies that in the oblique solid all nearest neighbor particles are not equidistant. There are two length scales present in the system, which is a consequence of the two length scale potential. Particles having lower bond length are stabilized by isotropic LJ interaction and larger bond lengths are stabilized by anisotropic HB interaction. Contrary to the oblique solid, honeycomb solid is stabilized only by anisotropic HB interactions and thus the nearest neighbor distance distribution has only one peak centered at HB length and there is no splitting in the first peak of radial distribution function.

In the subsequent sections, first, we shall discuss the temperature induced transition followed by a comparative study of pressure and temperature induced transitions.

\section{B. Order parameters and their susceptibilities}

In Fig. 3, we have shown the order parameters change and their susceptibilities for temperature induced collapse transition. On increasing temperature, thermal fluctuations destabilize the honeycomb structure and collapses to a high density oblique structure with large change in density (from $\sim 0.79$ to $\sim 1.56$ ) (see Fig. 3A). In the collapsed transition, high density change indicates that the transition can only be first order. As due to increase in order parameter difference the surface energy cost for formation of new phase also increases. Since the barrier in the global free energy surface as function of global order parameter (density and order) is related to the surface tension, the probability of long wavelength fluctuations of the global order parameters (density fluctuation is coupled with order fluctuation) in the system is less. In Fig. 3B, we have reported the change of both the global six-fold bond orientational order $\left(\psi_{6 g}\right)$ and local three-fold bond orientational order $\left(\psi_{3 l}\right)$ with temperature. $m$-fold global bond orientational order $\left(\psi_{m g}\right)$ is defined as

$$
\psi_{m g}=\frac{1}{N}\left\langle\left|\sum_{k=1}^{N}\left(\frac{1}{N N} \sum_{j=1}^{N N} e^{i m \theta_{k j}}\right)\right|\right\rangle,
$$

and $m$-fold local bond orientational order $\left(\psi_{m l}\right)$ is defined as

$$
\psi_{m l}=\frac{1}{N}\left\langle\sum_{k=1}^{N}\left(\left|\frac{1}{N N} \sum_{j=1}^{N N} e^{i m \theta_{k j}}\right|\right)\right\rangle,
$$

where $m=6$ for six-fold and $m=3$ for three-fold symmetry. $N N$ is the number of nearest neighbors, $\theta_{i j}$ is the angle of the bond that the $j^{t h}$ neighbor makes with the tagged $i^{t h}$ particle. Significantly large values of global order parameter, $\psi_{6 g}$, in both phases indicate that there is a global translation of either six-fold or three-fold bond orientational order. As $\psi_{6 g}$ does not distinguish between the six-fold and three-fold bond orientation order, we have also plotted the local three-fold bond orientational order (note that global three-fold bond orientational order is zero for both six-fold and three-fold symmetry). However, the later does not guarantee the long range translation of local bond orientational order. Sharp transition in $\psi_{3 l}$ and significantly large values of $\psi_{6 g}$ in both the phases indicate a one step transition from three-fold honeycomb lattice to six-fold (distorted) oblique solid phase.

In Fig. 3C and 3D, we have plotted the susceptibilities along density (isothermal compressibility, $\kappa_{T}$ ) and 

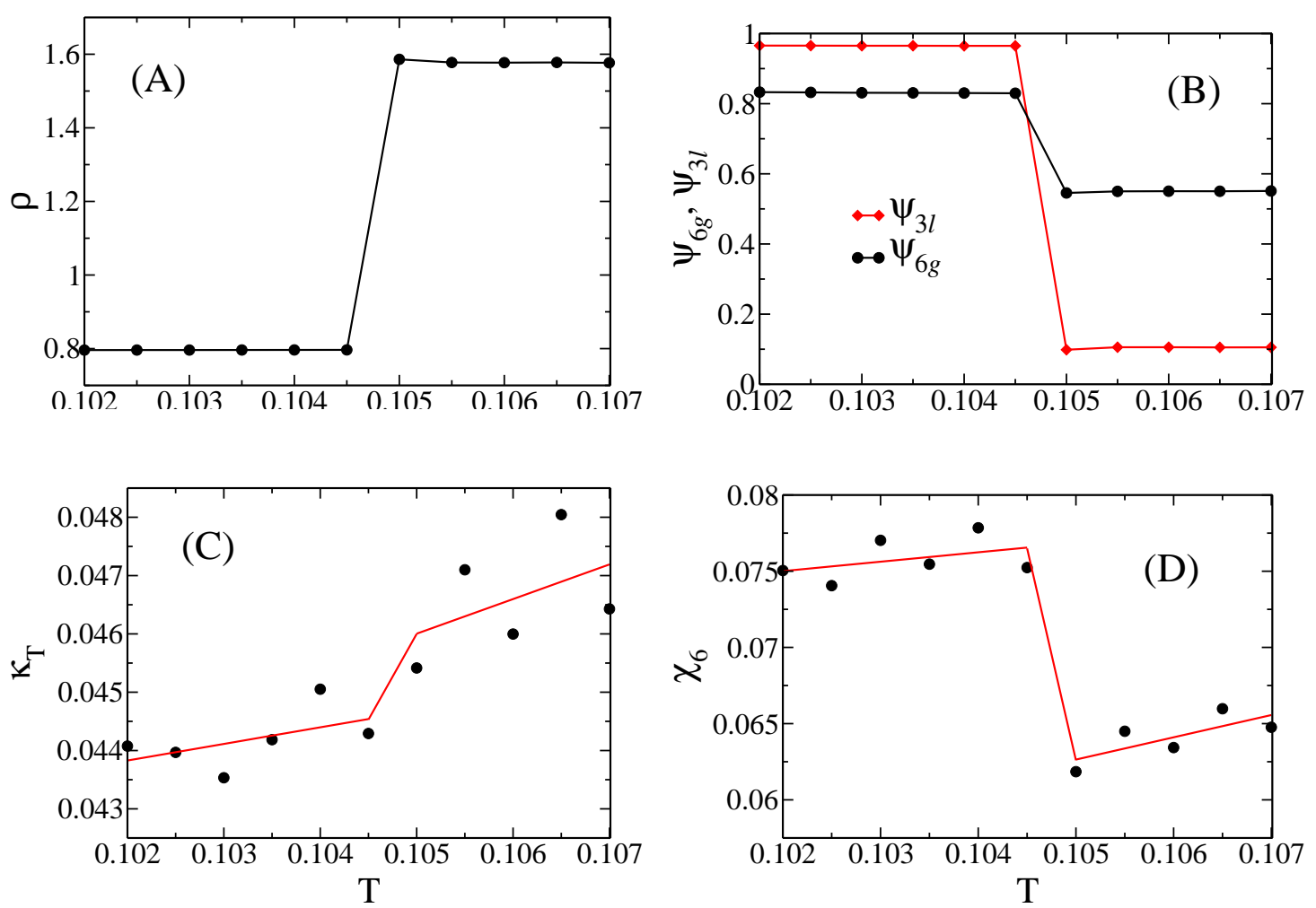

FIG. 3. (A) The dependence of the density $(\rho)$ of the system on temperature is shown. The starting configuration is honeycomb solid and after transition it goes to the oblique phase. (B) The dependence of global six-fold $\left(\psi_{6 g}\right)$ and local three fold $\left(\psi_{3 l}\right)$ bond orientational order parameters on temperature is shown. In (C) and (D), we have shown the dependence susceptibilities of global order parameters (density and six-fold bond orientational order parameter): isothermal compressibility $\left(\kappa_{T}\right)$ and sixfold bond orientational order susceptibility $\left(\chi_{6}\right)$ on temperature. Weak temperature dependence and finite jump in response functions suggests strongly $1^{\text {st }}$ order transition.

global six-fold bond orientational order parameter $\left(\chi_{6}\right)$. Six-fold bond orientational order susceptibility is defined as $\chi_{6}=\left\langle\left|\psi_{6 g}\right|^{2}\right\rangle-\left\langle\left|\psi_{6 g}\right|\right\rangle^{2}$ and and isothermal compressibility is defined in terms of volume fluctuations as $\kappa_{T}=\left(\left\langle V^{2}\right\rangle-\langle V\rangle^{2}\right) / T\langle V\rangle$. Weak temperature dependence and finite jump in both $\kappa_{T}$ and $\chi_{6}$ suggest the strongly $1^{\text {st }}$ order transition.

\section{Microscopic pathway of temperature induced collapse transition}

In order to find the pathway of transition first we need to distinguish the particles belonging to new phase in the sea of metastable bulk honeycomb solid phase. To distinguish the nucleating and growing new oblique-like particles from the parent metastable honeycomb phase, we computed the distribution of local 3 -fold bond orientational order $\left(\psi_{3 i}\right)$, defined as $\psi_{3 i}=\left|\sum_{j=1}^{N N} e^{i 3 \theta_{i j}} / N N\right|$, for both the solid phases. As shown in Fig. 4, the complete separation of two distributions enables us to separate the growing new phase from the parent honeycomb solid phase. Due to significant overlap in the distribu-

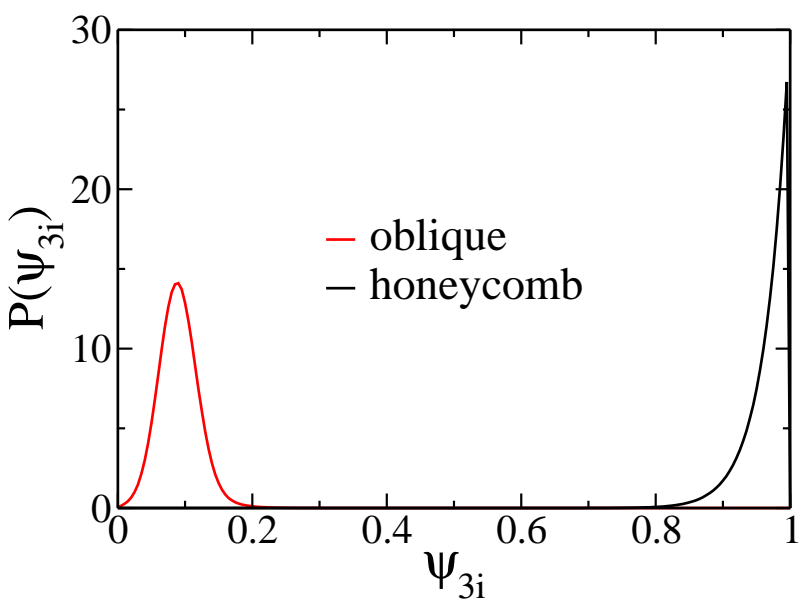

FIG. 4. Distributions of 3-fold local bond orientational order $\left(\psi_{3 i}\right)$ for honeycomb and oblique solids are shown.

tion of local six-fold bond orientational order we have not used it as a parameter to distinguish the two phases. Note that, though, $\psi_{3 i}$ is able to separate the oblique solid from the honeycomb solid phase, it does not distin- 

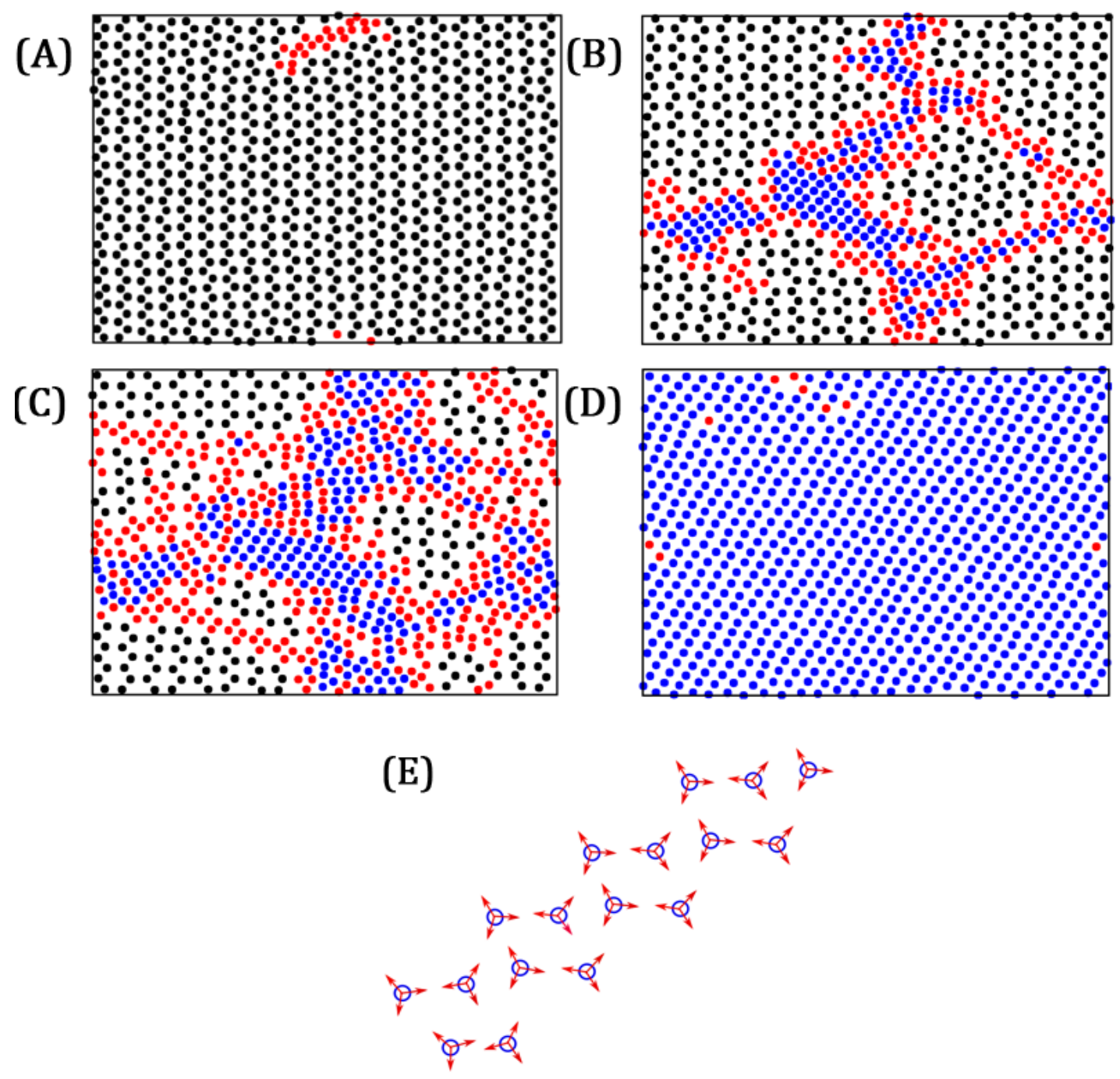

FIG. 5. Snapshots of the system at (A) early (B) intermediate and (C) late stage of transition are shown. (D) Oblique solid phase after transition for $N=836$ particles are shown. Note the linear strip-like arrangement transforming into branched and ring-like strips. Oblique solid has string-like arrangement of particles. We also note the wetting of oblique phase by a layer of intermediate solid phase. (E) Snapshot of a growing nucleus is shown.

guish the oblique solid-like and liquid-like particles (as the peak of distribution is centered at very low value of $\left.\psi_{3 i}\right)$. A two order parameter criteria using the values of both $\psi_{3 i}$ and $\psi_{6 i}$ is required to separate the oblique phase with liquid-like particles as the oblique solid is characterized with low $\psi_{3 i}$ and high $\psi_{6 i}$ values (see Fig. 3B).

In Fig. 5A - 5D, we have shown the snapshots of the system at different stages during the collapse transition. Depending on $\psi_{3 i}$ value we have defined three types of particles in the system. The particles having $\psi_{3 i}$ value greater than 0.75 is considered as honeycomb type and indicated with black color, red color particles have intermediate order with $\psi_{3 i}$ value between 0.25 and 0.75 . The particles having $\psi_{3 i}$ value less than 0.25 and $\psi_{6 i}$ value greater than 0.2 (lower cut-off value of $\psi_{6 i}$ below which the distribution of $\psi_{6 i}\left(P\left(\psi_{6 i}\right)\right)$ in oblique phase is zero) are considered as oblique-like. Note that these criteria do not distinguish oblique-like and triangular solid-like particles. However, simplicity of $2 \mathrm{D}$ systems allows us to distinguish these two types of orders by visual inspection of snapshots. Recently, Onuki et al. developed an elegant approach based on local bond orientational order parameter to quantify different phases, stacking faults and grain boundaries in both two and three dimensional systems 22, 23].

Contrary to the common believe and classical nucleation theories that the nuclei of new phase are circular in 2D) and have same order and density as of bulk stable phase, interestingly, we find highly anisotropic growth of new phase. At the early stage of growth, the cluster grows as linear strip (having significantly different order than the bulk oblique phase) followed by branched and ring-like strip and finally engulfs the whole system. The geometry of nucleating and growing cluster (shown in 

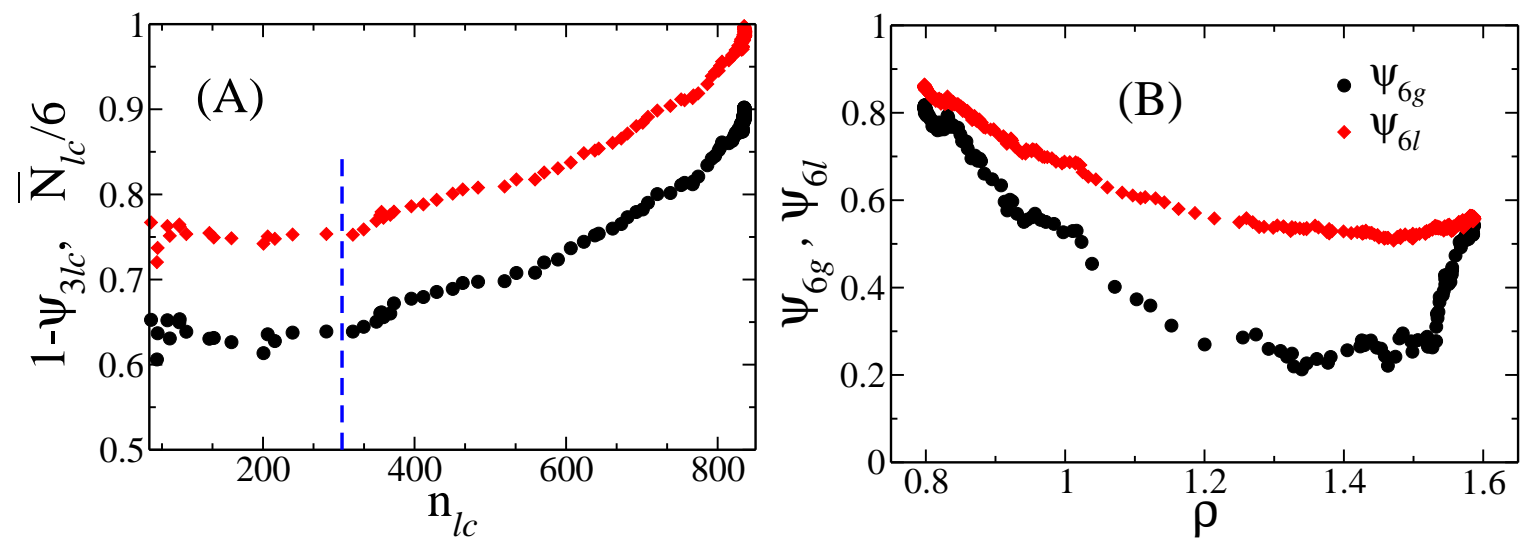

FIG. 6. (A) The dependence of local order parameter, 1- $\psi_{3 l_{c}}$ (black filled circles) and average coordination number scaled with six (the average coordination number of particles in oblique solid phase), $\bar{N}_{l c} / 6$ (red filled diamonds) of the largest cluster on the size of the largest cluster is shown. For convenience in comparison we have plotted $1-\psi_{3 l c}$ in place of $\psi_{3 l c}$. All quantities are averaged over $100 \mathrm{MC}$ steps along the growth trajectory for $N=836$ particle system. We have also observed similar trend for $N=1008$. The blue dotted line indicates the critical cluster size up to which there is no appreciable change in either average order or the coordination number of the cluster. (B) Pathways of solid-solid transition in two order parameter (density and six-fold bond orientational order) space are shown. The black filled circles represent the evolution of global six-fold bond orientational $\left(\psi_{6 g}\right)$ order with density and red filled diamonds represent the evolution of local six-fold bond orientational order $\left(\psi_{6 l}\right)$ with density.

Fig. 5E) is a consequence of delicate balance between the two (isotropic and anisotropic) types of interactions which enables the dominance of stabilizing energy over the destabilizing surface energy. Sharing of the H-bonds (see Fig. 5E) between the growing cluster and the bulk metastable honeycomb phase minimizes the surface energy cost for formation of new phase. We also observe that the structure of the growing cluster is different from the bulk oblique solid phase. As shown in Fig. 5B and $5 \mathrm{C}$, the oblique-like phase starts appearing inside the high density intermediate order cluster. There is no clear dividing surface between the oblique and the honeycomb and hence it is difficult to assign unambiguously the identity of particles as honeycomb or oblique-like. Wetting of the oblique-like phase by intermediate order particles reduces the surface free energy cost for the formation of stable oblique phase.

Thus, the solid-solid transition in a molecular system is a two step process. In the first step small anisotropic cluster having significantly different order nucleates and grows. Anisotropic shape enables the cluster to maximize the energy gain due to strong specific interactions among particles inside the cluster as well as with the bulk parent honeycomb phase. In the second step, due to internal rearrangement inside the dense (intermediate ordered) anisotropic cluster oblique-like phases start appearing. Wetting of nuclei by intermediate order phase is also observed in crystal nucleation from metastable liquid [24] where nucleation preferentially takes place in regions of high structural order. Note that clear dividing surface with no sharing of particles between the two phases (cluster of stable phase and bulk metastable phase) creates a density/order deficiency and thus increases the surface energy 25]. Wetting is one of the factors responsible for the observed discrepancy between the predictions of classical nucleation theory and experimental/simulation studies.

In Fig. 6A, we have shown the evolution of the average order and average coordination number of a growing cluster. We consider a particle not as honeycomb like if it has $\psi_{3 i}$ value less than 0.75 . Particles having $\psi_{3 i}$ value less than 0.75 and are connected by neighborhood form a cluster of new phase. The order of the cluster $\left(\psi_{3 l c}\right)$ is defined as, $\psi_{3 l c}=\sum_{i=1}^{n_{l c}} \psi_{3 i} / n_{l c}$, where $n_{l c}$ is the size of the largest cluster and summation is over all the particles that belong to the largest cluster. Average coordination number is scaled with the average coordination number of particles in the oblique solid phase. Since only one cluster grows in the system the largest cluster contains the maximum fraction of particles belonging to the new phase. The cluster of new phase has significantly different order from the bulk stable phase. During the growth, both the order and average coordination number of the cluster gradually evolve and finally merge to the value corresponding the bulk oblique solid phase. As shown in the figure, there is a crossover (indicated by blue dotted line in Fig. 6A) from weakly dependence to non-linear dependence of order as well as average coordination number on size of the cluster. At early stage of growth, weak dependence of the order and average coordination number on size of the cluster indicates the linear strip-like growth, where surface to volume ratio does not change appreciably. The non-linear dependence of order/average coordination number on cluster size is a consequence of 
two factors: (i) the change of surface to bulk ratio due to branching and swelling of the growing cluster and (ii) the rearrangement of the particles inside the cluster towards oblique-like structure.

In Fig. 6B, we have shown the evolution of both global six-fold bond orientational order $\left(\psi_{6 g}\right)$ and local six-fold bond orientational order $\left(\psi_{6 l}\right)$ of system with density during collapse transition. We observe an almost monotonic decrease of $\psi_{6 l}$ during transition from the value in the honeycomb to its value in the oblique solid phase. However, $\psi_{6 g}$ shows strongly non-monotonic evolution from its value in the honeycomb to the oblique solid phase. This non-monotonic evolution of $\psi_{6 g}$ and monotonic (or very weakly non-monotonic) evolution of $\psi_{6 l}$ arises due to cancellation of components of global bond orientational order at intermediate stage of growth and suggests the presence of domains (separated by grain boundaries) having different symmetry axes. Presence of domains having distinct symmetry axes does not alter the values of local order parameters. This scenario is also evident from the snapshots shown in Fig. 5. In the late stage of growth due to relaxation of grain boundaries the global order parameter value again increases and finally merges to the value corresponding to the bulk oblique phase.

\section{Does the pathway of pressure induced transition differ from temperature induced transition?}

Temperature plays two roles in the phase transition (i) it couples with entropy and perturbs the entire free energy landscape and (ii) it provides sufficient thermal energy to overcome the free energy barrier for phase transition. In the case of condensation and crystallization, where the entropy difference between the two phases is large, the dependence of free energy barrier on temperature is stronger than the case of solid-solid phase transitions. As discussed earlier, in the case of solid-solid transition where specific interactions play important role in the stability of both the phases we can assume that the major role of temperature is to provide sufficient thermal energy to overcome the free energy barrier for transition. As the honeycomb solid is metastable at a given initial pressure and temperature with sufficiently high free energy barrier, transition can take place in two ways - (i) increase the temperature until the thermal energy is sufficient to overcome the free energy barrier (this is called temperature induced transition and discussed earlier) and (ii) increase the pressure until the given initial thermal energy is sufficient to overcome the barrier. Increase of pressure favors high density phase and thus decreases the free energy barrier for transition. In this section we shall compare these two scenarios and discuss how the pathway of pressure induced transition at low temperature differ from temperature induced transition.

In Fig. 7 we have compared the pathways for pressure and temperature induced transitions in two dimensional order parameter (density and six-fold bond orientational order) space. For temperature induced transition the transition temperature is $T=0.105$ at $P=0.10$. In the case of pressure induced transition we have increased the pressure of the system (details are mentioned in Section II) at constant temperature $T=0.05$ and the observed transition pressure is $P \sim 0.45$. On increasing pressure transition temperature shifts towards lower temperature. In the case of pressure induced transition, we find that different trajectories collapse to different metastable disordered states (with slightly different density and order) and transition pressure also vary in orders of 0.01. As shown in the figure, contrary to the temperature induced transition where the honeycomb solid collapses to high density crystalline oblique solid phase, in the case of pressure induced transition the honeycomb solid collapses to a long lived disordered metastable state having very small global order parameter value (see Fig. 7A). Disordered metastable phase evolves towards the stable state with an extremely slow rate. In spite of the absence of the global six-fold bond orientational order, significantly large value of the local six-fold bond orientational order (see Fig. 7B) suggests the heterogeneous structure of the metastable phase where significant local six-fold symmetry is present but there is no global translation of the local order.

In order to gain more insight in the microscopic structure of the disordered solid phase, in Fig. 8, we have shown the distributions of local six-fold bond orientational order $\left(\psi_{6 i}\right)$ for both the oblique and the disordered solid phase. The emergence of the shoulder in the distribution corresponding to the disordered solid phase indicates the presence of heterogeneous local environments. To find the structures of different local environments responsible for the broadening of $\psi_{6 i}$ distribution towards the lower value, in inset we have shown the distributions of $\psi_{m i}\left(P\left(\psi_{m i}\right)\right)$ for the particles having coordination number $m$. That is we have computed the distribution of $\psi_{6 i}$ for only those particles which have coordination number six and so on. The distribution is normalized with the total number of particles so that area under curve of $P\left(\psi_{m i}\right)$ will give the fraction of particles having coordination number $m$. We note the presence of significant five-cordinated $(\sim 30 \%)$ and seven-coordinated $(\sim 18 \%)$ along with six-coordinated $(\sim 50 \%)$ particles with respective symmetries. On heating the metastable disordered solid phase undergoes a transition to the stable oblique phase having unimodal $\psi_{6 i}$ distribution. On increasing pressure the peak of the distribution of $\psi_{6 i}$ in the oblique solid phase shifts towards lower $\psi_{6 i}$ value. Recently, Kadau et al. [5] used molecular dynamics simulations to investigate the shock-induced phase transformation of solid iron. Above a critical shock strength the nucleation and growth become collective as many small grains start growing simultaneously. However, we have observed a single cluster growth scenario in both pressure and temperature induced transitions. Collective nucleation and growth scenario indicates the absence of free 

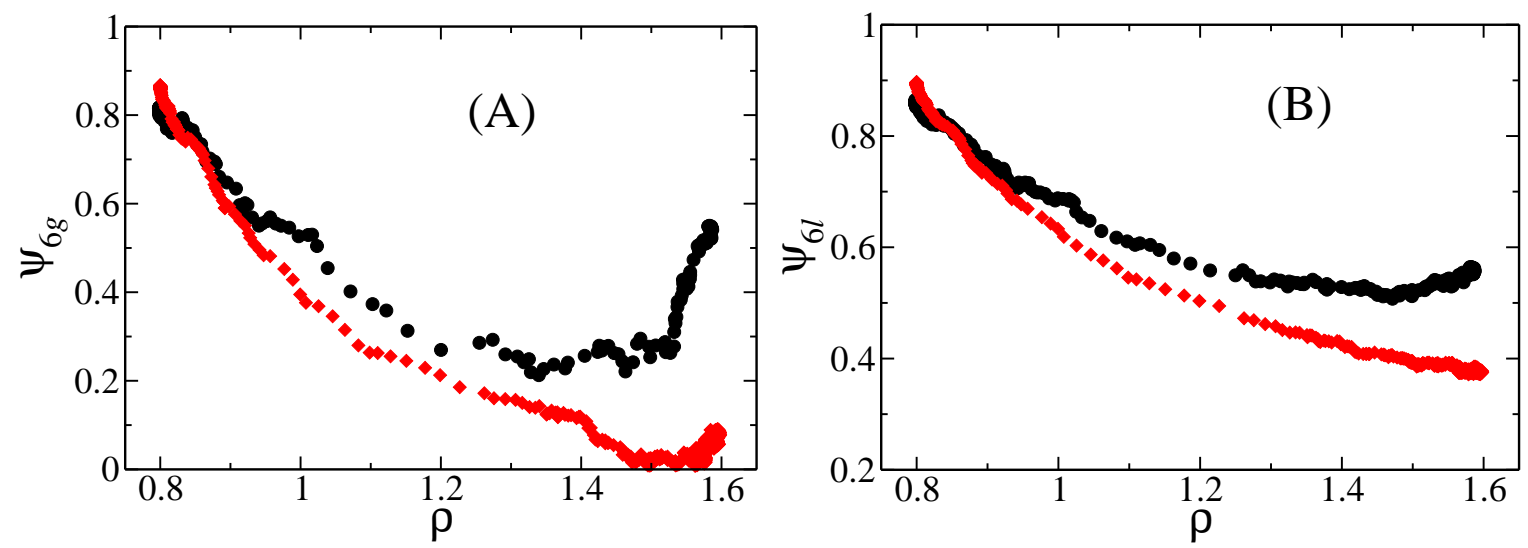

FIG. 7. Pathways for pressure and temperature induced transitions in two dimensional order parameter (density and global six-fold bond orientational order) space are shown. (A) density $(\rho)$ and global six-fold bond orientational order parameter $\left(\psi_{6 g}\right)$ space. (B) density $(\rho)$ and local six-fold bond orientational order parameter $\left(\psi_{6 l}\right)$ space. Black filled circles represent the pathway for temperature induced transition and red filled diamonds represent the pathway for pressure induced transition.

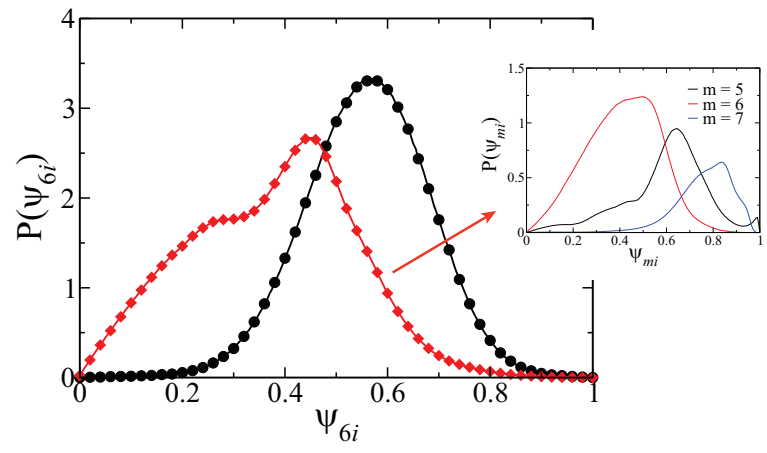

FIG. 8. Distributions of local six-fold bond orientational order $\left(\psi_{6 i}\right)$ for the collapsed structure in the case of temperature induced transition (black line distribution with filled circles) and pressure induced transition (red line distribution with filled diamonds) are shown. In case of temperature induced transition the collapsed structure is oblique solid and in the case of pressure induced transition the collapsed structure is disordered solid. In inset, distributions of $m$-fold local bond orientational order $\left(\psi_{m i}\right)$ of particles having coordination number $m$ for the pressure induced collapsed phase are shown. In the figure the distribution with black line indicates the $\psi_{5 i}$ distribution of particles having coordination number five, red line indicates $\psi_{6 i}$ distribution of particles having coordination number six and blue line indicates $\psi_{7 i}$ distribution of particles having coordination number seven. Area under $\psi_{m i}$ distribution gives the fraction of particles having coordination number $m$.

energy barrier for nucleation. Note that shock waves increase both the pressure as well as temperature and increment in both decreases the free energy barrier for transition. Above a critical shock strength the free energy barrier might be negligibly small and hence many clusters start growing simultaneously. This transition closely resembles with the gas-liquid transition at large metastabilty (above kinetic spinodal) where one observes similar collective nature of nucleation and growth 26 28]. In the case of pressure induced transition (or low temperature transition as on increasing pressure transition point shifts towards the lower temperature) trapping of the system in a long lived local minima indicates that the free energy landscape is rugged. Although one might observe the rugged energy landscape in atomic systems also, inclusion of specificity in interaction potential enhances the ruggedness of the landscape. The origin of enhanced ruggedness lies in the competition between specific and non-specific interactions where local regions might be energetically stabilized by a particular type of interaction but system as a whole might not be in global minimum. These local traps in the landscape decreases significantly the dynamics of the transition. On increasing temperature the disordered metastable solid phase undergoes a transition to the crystalline oblique phase as on increasing temperature system attains sufficient thermal energy to overcome local energy barriers and finally undergoes a transition to global free energy minimum.

\section{CONCLUSION}

In this work we have employed computer simulation techniques to study the solid-solid collapse transition in a model molecular system interacting via both isotropic as well as anisotropic interactions. In the case of temperature induced transition the metastable honeycomb solid collapses to high density crystalline oblique phase. High density oblique solid has two characteristic length scales corresponding to isotropic and anisotropic parts of interaction potential. Contrary to the common believe and classical nucleation theory that the nuclei of new phase are circular (in 2D) and has same order and 
density as of bulk stable phase, interestingly, we find linear strip-like nucleating clusters having significantly different order and average coordination number than the bulk stable oblique phase. Both the order and average coordination number of the growing cluster evolve with the size and finally converge to the order of the bulk stable phase. In the early stage of growth, the cluster grows as linear strip followed by branched and ring-like strips. Complex geometry of growing cluster is a consequence of the delicate balance between two types of interactions which enables the dominance of stabilizing energy over the destabilizing surface energy of the cluster. The nucleus of the stable oblique phase is wetted by a layer of intermediate order particles which minimizes the surface free energy cost for formation of the oblique phase. In the case of pressure induced transition the collapsed state is a disordered metastable solid. Pathways of pressure and temperature induced transitions are significantly different. These observations shed new light on the complex nature of nucleation and growth in molecular solid-solid transitions as well as on the validity of classical nucleation theories in complex molecular systems.

\section{ACKNOWLEDGMENTS}

We thank Prof. Surajit Sengupta and Dr. Mantu Santra for helpful discussions. We thank DST and BRNS, India for partial financial support of this work. B.B. thanks DST for a J.C. Bose fellowship.
[1] H. Czichos, The Martensitic Transformation in Science and Technology (DGM Informationsgesellschaft, Oberursel, Germany, 1989)

[2] G. B. Olson and W. Owen, Martensite (ASM International, Materials Park, OH, 1992)

[3] K. Otsuka and C. M. Wayman, Shape Memory Materials (Cambridge University Press, 1998)

[4] G. E. Duvall and R. A. Graham, Rev. Mod. Phys. 49, $523(1977)$

[5] K. Kadau, T. C. Germann, P. S. Lomdahl, and B. D. Holian, Science 296, 1681 (2002)

[6] M. H. Bocanegra-Bernal and S. D. D. la Torre, J. Mater. Sci. 37, 4947 (2002)

[7] K. Bhattacharya, S. Conti, G. Zanzotto, and J. Zimmer, Nature 428, 55 (2004)

[8] P. C. Hemmer and G. Stell, Phys. Rev. Lett. 24, 1284 (1970)

[9] D. A. Young and B. J. Alder, Phys. Rev. Lett. 38, 1213 (1977)

[10] S. V. Buldyrev, G. Malescio, C. A. Angell, N. Giovambattista, S. Prestipino, F. Saija, H. E. Stanley, and L. Xu, J. Phys.: Condens. Matter 21, 504106 (2009)

[11] A. M. Almudallal, S. V. Buldyrev, and I. Saika-Voivod, J. Chem. Phys. 137, 034507 (2012)

[12] S. Prestipino, F. Saija, and P. V. Giaquinta, J. Chem. Phys. 137, 104503 (2012)

[13] C. Mondal and S. Sengupta, Phys. Rev. E 84, 051503 (2011)
[14] P. Bolhuis, M. Hagen, and D. Frenkel, Phys. Rev. E 50, 4880 (1995)

[15] M. Rao and S. Sengupta, Phys. Rev. Lett. 91, 045502 (2003)

[16] M. Rao and S. Sengupta, Physica A 318, 251 (2003)

[17] M. Rao and S. Sengupta, J. Phys.: Condens. Matter 16, 7733 (2004)

[18] A. Ben-Naim, J. Chem. Phys 54, 3682 (1971)

[19] A. Ben-Naim, Mol. Phys 24, 705 (1972)

[20] K. A. T. Silverstein, A. D. J. Haymet, and K. A. Dill, J. Am. Chem. Soc 120, 3166 (1998)

[21] R. S. Singh, M. Santra, and B. Bagchi, J. Chem. Phys. (submitted)

[22] T. Hamanaka and A. Onuki, Phys. Rev. E 74, 011506 (2006)

[23] T. Kawasaki and A. Onuki, J. Chem. Phys. 135, 174109 (2011)

[24] T. Kawasaki and H. Tanaka, Proc. Nat. Acad. Sci. 107, $14036(2010)$

[25] F. Spaepen, Solid State Physics 47, 181 (1994)

[26] P. Bhimalapuram, S. Chakrabarty, and B. Bagchi, Phys. Rev. Lett. 98, 206104 (2007)

[27] M. Santra and B. Bagchi, Phys. Rev. E 83, 031602 (2011)

[28] M. Santra, R. S. Singh, and B. Bagchi, J. Stat. Phys. P03017 (2011) 